\title{
On the Integrated Density of States for Crystals with Randomly Distributed Impurities
}

\author{
Rainer Hempel $^{1 \star}$, Werner Kirsch ${ }^{2}$ \\ 1 Mathematisches Institut der Universität München, Theresienstr. 39, D-80333 München, \\ Germany \\ 2 Institut für Mathematik und Sonderforschungsbereich 237, Ruhr-Universität Bochum, \\ D-44780 Bochum, Germany
}

Received January 27, 1993

\begin{abstract}
In the present paper, we discuss spectral properties of a periodic Schrödinger operator which is perturbed by randomly distributed impurities; such operators occur as simple models for crystals (or semi-conductors) with impurities. While the spectrum itself is independent of the concentration $p$ of impurities, for $0<p<1$, we focus our attention on the limiting behavior of the integrated density of states $\rho_{p}$ of the random Schrödinger operator, inside a spectral gap of the periodic operator, as $p \rightarrow 0$. Denoting by $U_{0}$ the set of eigenvalues (in the gap) of the reference problem having precisely one impurity (located at the origin, say), we show that the integrated density of states concentrates around the points of $U_{0}$, in the sense that $\rho_{p}\left(U_{\varepsilon}\right)$ is of order $p$, for any fixed $\varepsilon$-neighborhood $U_{\varepsilon}$ of $U_{0}$, while $\rho_{p}(K) \leqq C \cdot p^{2}$, for any compact subset $K$ of the gap which does not intersect $U_{\varepsilon}$.
\end{abstract}

\section{Introduction}

We consider a simple model for a crystalline solid with impurities. In this model, atoms of a pure crystal are replaced by atoms of a different species (impurities) in a random way (i.e., at a lattice site there is an impurity with probability $p$ independent of the other sites). The spectrum $\Sigma$ of the resulting alloy can be described rather explicitly (see e.g. [KM1, EK, K1]). For any subset $I$ of the lattice $\mathbb{Z}^{v}$ let us denote by $\Sigma_{I}$ the spectrum of the crystal with impurities (exactly) at the sites $i \in I$. Then $\Sigma$, the spectrum of the random alloy, is (almost surely) given by:

$$
\Sigma=\overline{U \Sigma_{I}},
$$

where the union is extended over all finite subsets $I$ of $\mathbb{Z}^{v}$. Thus, $\Sigma$ consists of the spectrum of the pure crystal plus (the closure of) all the eigenvalues of systems with finitely many impurities. The closure of these eigenvalues will form, as a rule, bands

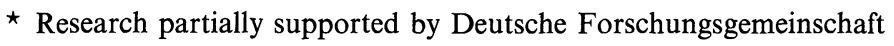


inside gaps of the pure material. These impurity bands are responsible for many interesting physical phenomena, e.g., color and light absorption of ruby and sapphire, and conductivity properties of semi-conductor devices (cf., e.g., [DH, H3, $\mathrm{KP}]$ and the physics literature quoted in these papers).

It follows from (1.1) that the spectrum $\Sigma$ of the crystal with impurities is independent of the concentration $p$ of the impurity atoms (for $0<p<1$ ) (see [EK, $\mathrm{K} 1])$. Thus, the spectrum remains constant if $p$ is changed, except possibly at $p=0$ (where the impurity bands collapse) and at $p=1$. While puzzling at a first glance, this result merely tells us that the spectrum is a very rough property of the disordered system.

More information can be obtained from the density of states measure $\rho$ of the system. Intuitively speaking $\rho(M),(M \subset \mathbb{R})$, measures how many "states" per unit cell of our system correspond to energies inside the set $M$. (We will give a precise definition of $\rho$ in the next section.) The support $\operatorname{supp} \rho$ of the density of states measure agrees with the spectrum $\Sigma$.

It can be shown that the density of states measure $\rho_{p}$ of the system with impurity concentration $p$ depends continuously on the parameter $p$ (in the sense of vague convergence of measures) (see $[\mathrm{K} 1, \mathrm{~K} 2]$ ). Consequently, the density of states of the impurity bands tends to zero as $p$ goes to zero. ${ }^{1}$ So, while the impurity bands still belong to the spectrum $\Sigma\left(=\operatorname{supp} \rho_{p}\right)$ they become less and less "densely filled".

In this paper we investigate more carefully, in which way the density of states $\rho_{p}(B) \equiv \rho(B)$ of an impurity band $B$ tends to zero as $p$ goes to zero. We prove that $\rho_{p}$ restricted to $B$ concentrates around $U_{0}$, the set of eigenvalues of the crystal with only one impurity. More precisely, we prove, that

$$
\rho_{p}\left(U_{\varepsilon}\right) \geqq C p
$$

if $U_{\varepsilon}$ is an $\varepsilon$-neighborhood of the set $U_{0}$ and that

$$
\rho_{p}\left(K \backslash U_{\varepsilon}\right) \leqslant C p^{2}
$$

if $K$ is any closed interval, whose intersection with the spectrum of the pure crystal is empty.

In Sect. 2 we give a precise statement of this result as well as an outline of the proof. Section 3 contains the main technical ingredient of our proof. We analyze the influence of a well separated impurity on the spectrum of Schrödinger operators on large (compact) boxes. This part of our investigation relies on work by Deift and Hempel $[\mathrm{H} 1, \mathrm{DH}]$ which contains exponential estimates on resolvent kernels.

The paper closes with two appendices where we collect some analytic and some probabilistic estimates.

We believe that the idea of our proof is quite lucid and, in fact, simple. To avoid obscuring these arguments we made no attempt to reach high generality in our assumptions.

\footnotetext{
1 The reader should be aware of the distinction between "alloys" on the one and "crystals with impurities" on the other side; in 3-dim. physical reality, the latter case will roughly correspond to $p<10^{-3}$
} 


\section{The Main Result}

Let $V$ be a $\mathbb{Z}^{v}$-periodic potential; for simplicity, we assume that $V$ is bounded. We denote by $H=H_{0}+V, H_{0}=-\Delta$ the Hamiltonian for the "pure crystal". By $w$ we denote the potential of a single impurity. We assume that $w$ is a continuous function with support inside the set $\left\{x \in \mathbb{R}^{v} \mid-1 / 2<x_{i}<1 / 2\right.$, for $\left.i=1, \ldots, v\right\}$. Thus the system with random impurities is described by the stochastic potential

$$
V_{\omega}(x)=V(x)+\sum_{i \in \mathbb{Z}^{v}} q_{i}(\omega) w(x-i),
$$

where we assume $q_{i}$ to be independent, identically distributed $\{0,1\}$-valued random variables. We write $H_{\omega}=H_{0}+V_{\omega}$. By $\mathbb{P}$ we denote the underlying probability measure and by $\mathbb{E}$ its corresponding expectation. The number $p=\mathbb{P}\left(q_{i}=1\right)$ gives the (relative) concentration of the impurity atoms.

We also define for any set $A \subset \mathbb{Z}^{v}$

$$
W_{A}(x)=\sum_{i \in A} w(x-i)
$$

as well as $V_{A}=V+W_{A}, H_{A}=H_{0}+V_{A}=H+W_{A}$.

To define the density of states measure for $H_{\omega}$ we have to restrict these operators to finite boxes. We denote by $Q_{N}=\left\{x \in \mathbb{R}^{\nu} \mid-N-\frac{1}{2} \leqq x_{i} \leqq N+\frac{1}{2}\right.$ for $i=1, \ldots, v\}$, the hypercube of side length $2 N+1$ around the origin. By $H_{\omega}^{(N)}$ we mean the operator $H_{\omega}$ restricted to $L^{2}\left(Q_{N}\right)$ with periodic boundary conditions at $\partial Q_{N} ; H^{(N)}, H_{A}^{(N)}$ etc. are defined accordingly. For any $\omega$, the operator $H_{\omega}^{(N)}$ has purely discrete spectrum bounded from below. We denote the (projection-valued) spectral measure of a self-adjoint operator $T$ by $P_{M}(T), M \subset \mathbb{R}$ any Borel-set, so that $\operatorname{dim} P_{M}\left(H_{\omega}^{(N)}\right)$ is just the number of eigenvalues of $H_{\omega}^{(N)}$ inside the set $M$, counted according to their multiplicity.

The mapping $M \rightarrow \operatorname{dim} P_{M}\left(H_{\omega}^{(N)}\right)$ is a point measure on $\mathbb{R}$. The density of states measure $\rho$ for the operator $H_{\omega}$ is then defined by

$$
\rho(M)=\lim _{N \rightarrow \infty}\left|Q_{N}\right|^{-1} \operatorname{dim} P_{M}\left(H_{\omega}^{(N)}\right),
$$

where $\left|Q_{N}\right|$ denotes the volume of $Q_{N}$, and the limit is taken in the sense of vague convergence of measures. It is well known that the above limit exists almost surely (see, e.g., [Pas, KM2]) and is non-random. The measure $\rho$ will, of course, depend on the distribution of the random variables $\left\{q_{i}\right\}$, i.e., on the concentration $p$ of the impurity. To stress this dependence we sometimes write $\rho_{p}$ for $\rho$.

It is known that $\rho$ can also be obtained from

$$
\rho_{p}(\cdot)=\lim _{N \rightarrow \infty}\left|Q_{N}\right|^{-1} \mathbb{E}_{p}\left(\operatorname{dim} P_{(\cdot)}\left(H_{\omega}^{(N)}\right)\right)
$$

(in the sense of vague topology) where $\mathbb{E}_{p}$ is the expectation with respect to the underlying probability measure $\mathbb{P}_{p}$. It follows that for bounded open sets $G$

$$
\rho_{p}(G) \leqq \liminf _{N \rightarrow \infty}\left|Q_{N}\right|^{-1} \mathbb{E}_{p}\left(\operatorname{dim} P_{G}\left(H_{\omega}^{(N)}\right)\right),
$$


while for bounded closed sets $F$

$$
\rho_{p}(F) \geqq \limsup _{N \rightarrow \infty}\left|Q_{N}\right|^{-1} \mathbb{E}_{p}\left(\operatorname{dim} P_{F}\left(H_{\omega}^{(N)}\right)\right) .
$$

For the rest of this paper we fix an interval $[a, b]$ inside a gap of the spectrum $\Sigma_{\emptyset}=\sigma(H)$ and set $K=[a, b]$. By $U_{0}$ we denote the (finite) set of eigenvalues of $H_{\{0\}}=H_{0}+V+w$ inside $[a, b]$ and we set $U_{\varepsilon}=\left\{\lambda \in \mathbb{R} \mid \operatorname{dist}\left(\lambda, U_{0}\right)<\varepsilon\right\}$. Without restriction, we may henceforth assume that $a, b \notin U_{0}$ and that $\varepsilon_{0}>0$ is so small that $U_{3 \varepsilon_{0}} \subset(a, b)$.

Now, we can formulate our main result:

Theorem I. For $0<\varepsilon<\varepsilon_{0}$ fixed, there exist constants $C_{1}>0$ and $C_{2}$ such that

$$
\begin{aligned}
\rho_{p}\left(\tilde{U}_{\varepsilon}\right) & \geqq C_{1} p, \\
\rho_{p}\left(K \backslash U_{\varepsilon}\right) & \leqq C_{2} p^{2},
\end{aligned}
$$

for $0<p<1 / 2$, where $\tilde{U}_{\varepsilon}$ denotes any component of $U_{\varepsilon}$.

Theorem I tells us that, as $p$ tends to zero, the density of states inside a gap $[a, b]$ is more and more concentrated around the eigenvalues of $H+w$. Note that our result also applies if the set of eigenvalues $U_{0}$ is empty. On the other hand, if we introduce an additional coupling constant $\lambda \in \mathbb{R}$, then one can produce eigenvalues of $H-\lambda w$ at any energy in the gap, by choosing suitable $\lambda$ 's (cf., e.g., [ADH, DH, GS]).

The idea of the proof is as follows: Take $N$ very large and fix $\omega$. An impurity at $i \in Q_{N}$ (corresponding to $q_{i}(\omega)=1$ ) will produce an eigenvalue inside $[a, b]$ close to $U_{0}$, provided there are no other impurities near $i$. Indeed, in this case an eigenfunction of $H+w(\cdot-i)$ is almost an eigenfunction to $H_{\omega}^{(N)}$, if $N$ is large, because, by assumption, these operators are very similar close to the point $i$ and the eigenfunction will be concentrated around this point.

To be more precise, let us define for any $\omega$ and $N$,

$$
A=A^{(N)}=\left\{i \in Q_{N} \cap \mathbb{Z}^{v} \mid q_{i}=1\right\}
$$

the set of impurities in $Q_{N}$. We equip $\mathbb{R}^{v}$ with the metric $|i|=\max _{\alpha=1, \ldots, v}\left|i_{\alpha}\right|$ and set $\operatorname{dist}(x, M)=\inf _{y \in M}|x-y|$.

We define the set of $L$-isolated points in $A^{(N)}$ by:

$$
A_{L}=A_{L}^{(N)}=\left\{i \in A^{(N)} \mid \operatorname{dist}\left(i, A^{(N)} \backslash\{i\}\right)>L \text { and } \operatorname{dist}\left(i, \partial Q_{N}\right)>L\right\} .
$$

In Sect. 3 we prove the following theorem:

Theorem II. For $0<\varepsilon<\varepsilon_{0}$ fixed, there exists $L_{\varepsilon}$ such that, for any $\omega$ and any component $\tilde{U}_{\varepsilon}$ of $U_{\varepsilon}$

$$
\begin{aligned}
\operatorname{dim} P_{\tilde{U}_{\varepsilon}}\left(H_{\omega}^{(N)}\right) & \geqq \# A_{L}^{(N)}, \\
\operatorname{dim} P_{K \backslash U_{\varepsilon}}\left(H_{\omega}^{(N)}\right) & \leqq C\left(\# A^{(N)}-\# A_{L}^{(N)}\right)
\end{aligned}
$$

for $L \geqq L_{\varepsilon}$ and $N$ large enough (here $A^{(N)}=A^{(N)}(\omega)$, etc), where the constant $C$ is independent of $N, L$ and $\omega$.

Next, we estimate the expected number of (isolated) impurities. The following lemma will be proven in Appendix B: 
Lemma 2.1. (i) $\mathbb{E}\left(\# A^{(N)}\right)=\left|Q_{N}\right| \cdot p$.

$$
\text { (ii) } \mathbb{E}\left(\# A_{L}^{(N)}\right)=\left|Q_{N}\right| \cdot p(1-p)^{(2 L+1)^{\nu}}+O\left(N^{v-1}\right) \text {. }
$$

Given this lemma and Theorem II, we can now prove our main result: Proof of Theorem I.

$$
\begin{aligned}
\rho_{p}\left(\tilde{U}_{\varepsilon}\right) & \geqq \varlimsup_{N \rightarrow \infty}\left|Q_{N}\right|^{-1} \mathbb{E}_{p}\left(\operatorname{dim} P_{\tilde{U}_{\varepsilon}}\left(H_{\omega}^{(N)}\right)\right) \\
& \geqq \varlimsup_{N \rightarrow \infty}\left|Q_{N}\right|^{-1} \mathbb{E}_{p}\left(\# A_{L}^{(N)}\right) \\
& =C_{L} \cdot p
\end{aligned}
$$

where we took $L \geqq L_{\varepsilon}$.

(ii)

$$
\begin{aligned}
\rho_{p}\left(K \backslash U_{\varepsilon}\right) & \leqq \underline{\lim }_{N \rightarrow \infty}\left|Q_{N}\right|^{-1} \mathbb{E}_{p}\left(\operatorname{dim} P_{K \backslash U_{\varepsilon}}\left(H_{\omega}^{(N)}\right)\right) \\
& \leqq C \cdot \underline{\lim }_{N \rightarrow \infty}\left|Q_{N}\right|^{-1} \mathbb{E}_{p}\left(\# A^{(N)}-\# A_{L}^{(N)}\right) \\
& \leqq C\left\{1-(1-p)^{(2 L+1)^{\nu}}\right\} \cdot p \\
& \leqq C(2 L+1)^{v} \cdot p^{2} \\
& \leqq \tilde{C}_{L} p^{2}
\end{aligned}
$$

(where we use $(1-x)^{n} \geqq 1-n \cdot x$ ).

\section{Eigenvalue Estimates}

In this section we prove Theorem II, which is split into two pieces (Proposition 3.1 and 3.3). For the proofs we will need cutoff-functions $\psi_{L}$ defined in the following way: Let $\psi \in C_{c}^{\infty}\left(\mathbb{R}^{v}\right)$ with the property that $0 \leqq \psi \leqq 1$ and that $\psi(x)=1$, if $|x|<1 / 4$, and $\psi(x)=0$, if $|x| \geqq 1 / 2$. We then define

$$
\psi_{k}(x):=\psi(x / k), \quad x \in \mathbb{R}^{v}, \quad k>0 .
$$

In our first proposition we show that any $L$-isolated point of $A$ accounts for at least one eigenvalue of $H_{A}^{(N)}$ in each component of $U_{\varepsilon}$, provided $L$ is sufficiently large.

Proposition 3.1. For any $0<\varepsilon<\varepsilon_{0} \leqq 1 / 2$, there exists $L_{\varepsilon} \in \mathbb{N}$ such that the following is true: for any $A \subset \mathbb{Z}^{v} \cap Q_{N}$, the operator $H_{A}^{(N)}$ has at least \# $A_{L}^{(N)}$ eigenvalues (counting multiplicities) in each component $\tilde{U}_{\varepsilon}$ of $U_{\varepsilon}$, provided $L \geqq L_{\varepsilon}$. In particular, for any $\omega$ we have (cf. Theorem II, (i))

$$
\operatorname{dim} P_{\tilde{U}_{\mathrm{e}}}\left(H_{\omega}^{(N)}\right) \geqq \# A_{L}^{(N)}(\omega), \quad L \geqq L_{\varepsilon} .
$$

Remark. The proof shows that we may take $L_{\varepsilon} \sim \ln \varepsilon$, for $\varepsilon$ small.

Proof. Let $E \in U_{0}$ and let $u \in \mathscr{D}(H)$ satisfy $(H+w) u=E u,\|u\|=1$. With $\psi_{L}$ as defined above, we let $j_{L}:=\left\{x \in \mathbb{R}^{v} ;|x| \geqq L / 4\right\}$, so that, in particular, $\operatorname{supp} \nabla \psi_{L} \subset j_{L}$. To ensure $\psi_{L} w=w$, we'll only consider $L \geqq 2$ in the sequel. From

$$
\chi_{j_{L}} u=-\chi_{j_{L}}(H-E)^{-1} w u
$$


we may now conclude with the aid of Lemma 4.3, that

$$
\begin{aligned}
\left\|\chi_{j_{L}} u\right\| & \leqq\left\|\chi_{j_{L}}(H-E)^{-1} \chi_{\text {supp } w}\right\| \cdot\|w\|_{\infty} \cdot\|u\| \\
& \leqq C e^{-\alpha L}\|w\|_{\infty},
\end{aligned}
$$

for suitable constants $C$ and $\alpha>0$; hence $\left\|\psi_{L} u\right\|>1-\varepsilon / 2$, for $L$ sufficiently large. On the other hand, we have for $L \geqq 2$ that

$$
(H+w-E)\left(\psi_{L} u\right)=-2 \nabla \psi_{L} \cdot \nabla u-\Delta \psi_{L} u,
$$

where Lemma 4.4 yields a constant $d$, which may be chosen to be independent of $L \geqq 2$, such that

$$
\left\|\nabla \psi_{L} \cdot \nabla u\right\| \leqq d\left\|u \mid \operatorname{supp} \nabla \psi_{L}\right\| \leqq d\left\|\chi_{j_{L}} u\right\| .
$$

Combined with (3.1) it easily follows that

and, finally, that

$$
\left\|(H+w-E)\left(\psi_{L} u\right)\right\| \leqq C^{\prime} e^{-\alpha L},
$$

$$
\left\|(H+w-E)\left(\psi_{L} u\right)\right\| \leqq \varepsilon\left\|\psi_{L} u\right\|,
$$

for $L \geqq L_{\varepsilon} \geqq 2$. We now consider the translates $\left(\psi_{L} u\right)(\cdot-a), a \in A_{L}$, and define $M$ to be the subspace of $\mathscr{D}\left(H^{(N)}\right)$ which is spanned by these translates. The functions $\left(\psi_{L} u\right)(\cdot-a), a \in A_{L}$, have mutually disjoint supports, and therefore it is immediately clear that $\operatorname{dim} M=\# A_{L}$ and that $\left\|\left(H+W_{A}-E\right) v\right\|<\varepsilon\|v\|, v \in M$. Now the spectral theorem implies that

$$
\operatorname{dim} P_{(E-\varepsilon, E+\varepsilon)}\left(H_{A}^{(N)}\right) \geqq \operatorname{dim} M=\# A_{L},
$$

and we are done.

If all impurities in a given configuration $A$ are $L$-isolated (so that $A=A_{L}^{(N)}$ ), then $H_{A}^{(N)}$ has $n o$ eigenvalues in $K \backslash U_{\varepsilon}$, for $L$ sufficiently large:

Proposition 3.2. For $0<\varepsilon<\varepsilon_{0}$, there exists $L_{\varepsilon}^{\prime} \in \mathbb{N}$ such that $H_{A}^{(N)}$ has no spectrum in $K \backslash U_{\varepsilon}$ for all $A \subset \mathbb{Z}^{v} \cap Q_{N}$ which satisfy $A=A_{L_{\ell}}^{(N)}$.

Proof. Let $0<\varepsilon<\varepsilon_{0}$ be fixed, and suppose that $A$ is a configuration such that $A=A_{L}^{(N)}$, for some $L \geqq 2$.

Suppose $E \in K \backslash U_{\varepsilon}$ is an eigenvalue of $H_{A}^{(N)}$, with eigenfunction $u,\|u\|=1$. Choose $a^{*} \in A$ such that $\left\|W_{A} u \mid Q_{0}\left(a^{*}\right)\right\|$ is maximal, in the sense that

$$
\left\|W_{A} u\left|Q_{0}\left(a^{*}\right)\|\geqq\| W_{A} u\right| Q_{0}(a)\right\|, \quad a \in A ;
$$

here $Q_{r}(b)$ denotes the (standard) cube of sidelength $2 r+1$, centered at the point $b \in \mathbb{R}^{v}$; in particular, supp $w \subset Q_{0}(0)$.

We now apply the cut-off procedure from the proof of Proposition 3.1 around the point $a^{*}$. Letting

$$
\mathscr{T}:=\mathscr{T}_{L}:=\left\{x \in \mathbb{R}^{v} ; L / 4<\left|x-a^{*}\right|<L / 2\right\}, \quad L \geqq 2,
$$

we first show that there exist $c, \alpha>0$ such that $\left(\chi^{*}\right.$ denoting the characteristic function of the cube $Q_{0}\left(a^{*}\right)$ )

$$
\left\|\chi_{T} u\right\| \leqq c e^{-\alpha L}\left\|W_{A} u \mid Q_{0}\left(a^{*}\right)\right\| \leqq c e^{-\alpha L}\left\|\chi^{*} u\right\| \cdot\|w\|_{\infty}, \quad L \geqq 2 .
$$

In fact, from $u=-\left(H^{(N)}-E\right)^{-1} W_{A} u$, we get

$$
\chi_{\mathscr{T}} u=-\chi_{\mathscr{T}}\left(H^{(N)}-E\right)^{-1} W_{\left\{a^{*}\right\}} u-\chi_{\mathscr{T}}\left(H^{(N)}-E\right)^{-1} W_{A \backslash\left\{a^{*}\right\}} u .
$$


Applying Lemma 4.3 (with $R=1 / 2, M=L / 4$ ) to the first term on the RHS, we obtain a contribution smaller than $c e^{-\alpha L}\left\|\chi^{*} u\right\|$. Next, we consider the "layers"

$$
\mathscr{L}_{s}:=\left\{x \in \mathbb{R}^{v} ; s-1 \leqq\left|x-a^{*}\right|<s\right\}
$$

where, for any $s \in \mathbb{N}$, the $s^{\text {th }}$ layer $\mathscr{L}_{s}$ will contain at most $s^{v}-(s-1)^{v}$ elements of $A$ (a very crude estimate!), so that

$$
\left\|W_{A} u \mid \mathscr{L}_{s}\right\| \leqq c s^{v-1}\left\|\chi^{*} u\right\| \cdot\|w\|_{\infty},
$$

by the maximality property of $a^{*}$. Since the region $\left\{x \in \mathbb{R}^{v} ; 0<\left|x-a^{*}\right|<L\right\}$ is free of points of $A$, Lemma 4.3 implies that the second term on the RHS can be estimated by

$$
C \sum_{s=L}^{2 N} e^{-\alpha(s-L / 2)} s^{\nu-1}\left\|\chi^{*} u\right\| \cdot\|w\|_{\infty}
$$

proving (3.2).

Now let $\psi_{L}$ as above, and define $\psi_{L ; a^{*}}$ by

$$
\psi_{L ; a^{*}}(x):=\psi_{L}\left(x-a^{*}\right), \quad x \in \mathbb{R}^{v} .
$$

Then $\psi_{L ; a^{*}} u \in \mathscr{D}(H)$ and, for $L \geqq L_{\varepsilon} \geqq 2$,

$$
\begin{aligned}
\left\|\left(H+w\left(\cdot-a^{*}\right)-E\right)\left(\psi_{L ; a^{*}} u\right)\right\| & \leqq 2\left\|\nabla \psi_{L ; a^{*}} \cdot \nabla u\right\|+\left\|\Delta \psi_{L ; a^{*}} u\right\| \\
& \leqq c\left\|u\left|\mathscr{T}_{L}\left\|\leqq C e^{-\alpha^{\prime} L}\right\| u\right| Q_{0}\left(a^{*}\right)\right\| \\
& \leqq C e^{-\alpha^{\prime} L}\left\|\psi_{L ; a^{*}} u\right\|,
\end{aligned}
$$

by (3.2) and Lemma 4.4, where $C$ and $\alpha^{\prime}>0$ are suitable constants. Now the spectral theorem implies that $H+w$ must have an eigenvalue in the interval $\left(E-C e^{-\alpha^{\prime} L}, E+C e^{-\alpha^{\prime} L}\right) \subset(E-\varepsilon, E+\varepsilon)$, for $L \geqq L_{\varepsilon}$. This leads to the desired contradiction, since $E$ has distance at least $\varepsilon$ from $\sigma(H)$, by assumption.

We finally show that clustered impurities can produce at worst $C \cdot \#\left(A \backslash A_{L}\right)$ eigenvalues outside $U_{2 \varepsilon}$.

Proposition 3.3. For $0<\varepsilon<\varepsilon_{0}$, there exists $L_{\varepsilon}^{\prime \prime} \in \mathbb{N}$ with the following property: for any $A \subset \mathbb{Z}^{v} \cap Q_{N}$, the number of eigenvalues of $H_{A}^{(N)}$ in $K \backslash U_{2 \varepsilon}$ is bounded by $C \cdot \#\left(A \backslash A_{L}^{(N)}\right)$, for $L \geqq L_{\varepsilon}^{\prime \prime}$ with a constant $C=C_{\varepsilon}$ which may be chosen independent of $N, L$ and $A$. In particular, we have (cf. Theorem II (ii))

$$
\operatorname{dim} P_{K \backslash U_{2_{\varepsilon}}}\left(H_{\omega}^{(N)}\right) \leqq C \cdot\left(\# A^{(N)}(\omega)-\# A_{L}^{(N)}(\omega)\right), \quad L \geqq L_{\varepsilon}^{\prime \prime} .
$$

Proof. Let $0<\varepsilon<\varepsilon_{0}$ be given, and let $B:=A_{L}^{(N)}$. By Proposition 3.2, the operator $H^{(N)}+W_{B}$ has no eigenvalues in $K \backslash U_{\varepsilon}$, for $L \geqq L_{\varepsilon}^{\prime}$. We now consider the selfadjoint operator family

$$
H^{(N)}+W_{B}+\mu W_{A \backslash B}, \quad \mu \in \mathbb{R},
$$

and apply the Birman-Schwinger principle in order to obtain a bound on the number of eigenvalue branches crossing the levels $\partial U_{2 \varepsilon}$ and $\partial K$, while $\mu$ increases from 0 to 1 . Writing

$$
S_{\varepsilon}:=\partial U_{2 \varepsilon} \cup\{a+\varepsilon, b-\varepsilon\},
$$

a finite set, we note that any $E \in S_{\varepsilon}$ satisfies $\operatorname{dist}(E, \sigma(H))>\varepsilon$, and, by Proposition 3.2, also $\operatorname{dist}\left(E, \sigma\left(H_{B}^{(N)}\right)\right)>\varepsilon$. 
Now suppose that an eigenvalue branch of $H^{(N)}+W_{B}+\mu W_{A \backslash B}, \mu \geqq 0$, crosses a level $E \in S_{\varepsilon}$ at some $\mu \in(0,1]$. By the Birman-Schwinger principle, this implies that $\mu^{-1}$ is an eigenvalue (of the same multiplicity) of the associated BirmanSchwinger-kernel

$$
K_{A ; B}(E):=W_{A \backslash B}^{1 / 2}\left(H^{(N)}+W_{B}-E\right)^{-1}\left|W_{A \backslash B}\right|^{1 / 2},
$$

where, as usual, $W_{A \backslash B}^{1 / 2}:=\left(\operatorname{sign} W_{A \backslash B}\right)\left|W_{A \backslash B}\right|^{1 / 2}$. We therefore see that the number of eigenvalue branches which cross $E \in S_{\varepsilon}$ (counting multiplicities), is bounded from above by the number of real eigenvalues $\geqq 1$ of the kernel $K_{A ; B}(E)$. As a consequence, the number of eigenvalues of $H^{(N)}+W_{A}$ in $K \backslash U_{2 \varepsilon}$ is bounded by

$$
\sum_{E \in S_{\varepsilon}} \sum_{k}\left|\lambda_{k}\right|^{r} \leqq \sum_{E \in S_{\varepsilon}} \sum_{k} \mu_{k}^{r}=\sum_{E \in S_{z}}\left\|K_{A ; B}(E)\right\|_{\mathscr{B}_{r}}^{r}, \quad r \geqq 1,
$$

where $\lambda_{k}$ and $\mu_{k}$ denote the eigenvalues and singular values of $K_{A ; B}(E)$, respectively; in the first inequality, we have used the Schur-Lelesco-Weyl theorem (cf. [S1], [RS IV]). Below, we shall derive the estimate

$$
\left\|K_{A ; B}(E)\right\|_{\mathscr{B}_{r}}^{r} \leqq C_{\varepsilon}(r)(\# A-\# B), \quad E \in S_{\varepsilon},
$$

for $r>v / 2$, and the desired result follows.

For a proof of (3.4), we use the second resolvent equation to obtain

$$
\begin{aligned}
& \left(H^{(N)}+W_{B}-E\right)^{-1} \\
& \quad=\left(H_{\delta}^{(N)}+1\right)^{-1}-\left(H_{0}^{(N)}+1\right)^{-1}\left[V+W_{B}-E-1\right]\left(H^{(N)}+W_{B}-E\right)^{-1},
\end{aligned}
$$

where $\left\|V+W_{B}-E-1\right\| \leqq\|V\|_{\infty}+\|w\|_{\infty}+E+1$, and $\left\|\left(H^{(N)}+W_{B}-E\right)^{-1}\right\|$ $<1 / \varepsilon$. Therefore, it is clearly enough to produce an estimate

$$
\left\|\left(H_{0}^{(N)}+1\right)^{-1} W_{A \backslash B}^{1 / 2}\right\|_{\mathscr{B}_{r}}^{r} \leqq C^{\prime} \#(A \backslash B),
$$

where the constant $C^{\prime}$ depends on $r$ only. We now fix some $q \in \mathbb{N}$ such that $r:=2^{q}>v / 2$, and employ Lemma 4.5 to obtain

$$
\left\|\left(H_{0}^{(N)}+1\right)^{-1} W_{A \backslash B}^{1 / 2}\right\|_{\mathscr{B}_{r}}^{r} \leqq \operatorname{trace}\left(\chi_{\operatorname{supp} W_{A \backslash B}}\left(H_{0}^{(N)}+1\right)^{-r} \chi_{\text {supp } W_{A \backslash B}}\right) \cdot\|w\|_{\infty}^{r / 2} .
$$

By Lemma 4.1, the kernel of $\left(H_{0}^{(N)}+1\right)^{-r}$ is uniformly bounded, and the desired inequality (3.5) follows.

\section{Appendix A: Auxiliary Analytic Results}

In this appendix, we first discuss several basic properties of the integral kernels associated with the operators $H_{0}^{(N)}$ and $H^{(N)}$. Throughout this section, $\chi_{n}$ will denote the characteristic function of the hypercube $Q_{n}$.

Lemma 4.1. For $m>v / 2$, the operator $\left(H_{0}^{(N)}+1\right)^{-m}$ has a continuous integral kernel $k_{m}^{(N)}(x, y)$, and there exists a constant $C$ such that $0 \leqq k_{m}^{(N)}(x, y) \leqq C$, for $x, y \in Q_{N}, N \in \mathbb{N}$.

Proof. Letting $f_{m^{\prime}}$ denote the Fourier transform of $\left(1+|\xi|^{2}\right)^{-m^{\prime}}$, for $m^{\prime} \in \mathbb{N}$, we know that $(-\Delta+1)^{-m^{\prime}}$ is just convolution with the function $f_{m^{\prime}}$ and that the 
$f_{m^{\prime}}$ decay rapidly away from 0 . Proceeding as in Courant and Hilbert [CH; Sect. V.15], we now observe that the integral kernel of $\left(H_{0}^{(N)}+1\right)^{-1}$ is given by

$$
R(x, y)=\chi_{N}(x) \sum_{a \in \Lambda_{N}} f_{1}(x-y-a) \chi_{N}(y),
$$

where $\Lambda_{N}:=(2 N+1) \mathbb{Z}^{v}$; this follows from the fact that, for $\varphi \in C_{c}^{\infty}\left(Q_{N}\right)$,

$$
\sum_{a \in \Lambda_{N}}(-\Delta+1)^{-1} \varphi(\cdot-a)
$$

defines a smooth function $F$ on $\mathbb{R}^{v}$ which is periodic with respect to the lattice $\Lambda_{N}$ and which clearly satisfies $(-\Delta+1) F=\varphi$ on $Q_{N}$.

Hence, $F$ belongs to the form domain (and even to the operator domain) of $H_{0}^{(N)}$, and satisfies $\left(H_{0}^{(N)}+1\right) F=\varphi$. By the above, we have $\chi_{N} F=\int R(x, y) \varphi(y) d y$. Therefore, the inverse of $H_{0}^{(N)}+1$ and the integral operator generated by $R$ coincide on $C_{c}^{\infty}\left(Q_{N}\right)$.

Now an easy calculation (using $f_{p} * f_{q}=f_{p+q}$ ) shows that the integral kernel of $\left(H_{0}^{(N)}+1\right)^{-m}$ is given by

$$
\chi_{N}(x) \sum_{a \in \Lambda_{N}} f_{m}(x-y-a) \chi_{N}(y) ;
$$

but, as remarked above, for $m>v / 2$, the function $f_{m}$ is bounded, continuous and decays rapidly away from the origin, and the desired result follows.

The following exponential decay estimate is basic for most of our analysis:

Lemma 4.2. Let $V: \mathbb{R}^{v} \rightarrow \mathbb{R}$ be continuous and periodic with respect to the lattice $\mathbb{Z}^{v}$ and let $H=-\Delta+V$. Then, for any $E \in \mathbb{R} \backslash \sigma(H)$ there exist constants $C, \alpha>0$, such that

$$
\left\|\chi_{R}(H-E)^{-1}\left(1-\chi_{M}\right)\right\| \leqq C e^{-\alpha(M-R)}, \quad 1 \leqq R<M / 2 .
$$

A proof of this basic lemma may be found, e.g., in Deift and Hempel [DH] or Hempel [H1, H2].

Lemma 4.3. Let $V: \mathbb{R}^{v} \rightarrow \mathbb{R}$ be continuous and periodic with respect to the lattice $\mathbb{Z}^{\nu}$. Let $H^{(N)}=-\Delta^{(N)}+V$ on $Q_{N}$, with periodic boundary conditions, and let $E \in \mathbb{R} \backslash \sigma(H)$. Then $E \notin \sigma\left(H^{(N)}\right)$ and there exist constants $C, \eta>0$, which are independent of $N, M$ and $R$, such that

$$
\left\|\chi_{R}\left(H^{(N)}-E\right)^{-1}\left(1-\chi_{M}\right) \chi_{N}\right\| \leqq C e^{-\eta(M-R)}, \quad 1 \leqq R<M / 2, \quad M \leqq N .
$$

Proof. As in the case of Lemma 4.1 we may write ( $\tau_{a}$ denoting translation by the vector $a$, i.e., $\left.\left(\tau_{a} \varphi\right)(x):=\varphi(x-a)\right)$

$$
\left(H^{(N)}-E\right)^{-1}=\chi_{N} \sum_{a \in \Lambda_{N}}(H-E)^{-1} \tau_{a} \chi_{N} ;
$$

using Lemma 4.2 it is clear that the sum converges in operator norm and that the desired estimate holds; note that for $z \in \Lambda_{N}, z \neq 0$, we have the trivial estimate $\operatorname{dist}\left(\tau_{z} Q_{N}, Q_{R}\right) \geqq\left(\max _{i=1, \ldots, v}\left|z_{i}\right|\right) \cdot N-R$.

We conclude this section with two simple but useful estimates. 
Lemma 4.4. (cf. [S2; Lemma C.2.1]) Let $\Omega$ be an open set in $\mathbb{R}^{v}$, let $U \in L_{\infty}(\Omega)$ and suppose that $u$ is a distributional solution of $(-\Delta+U) u=0$ in $\Omega$. Then, for any $\psi \in C_{c}^{\infty}(\Omega)^{v}$, there exists a constant $d$, such that

$$
\|\psi \cdot \nabla u\| \leqq d\|u \mid \operatorname{supp} \psi\|,
$$

where d depends on the sup-norms of $U, \psi$ and of $\Delta\left(|\psi|^{2}\right)$ only.

Lemma 4.5. (cf. Hempel [H3; Lemma 1.2]) Let $q \in \mathbb{N}$ and $r=2^{q}$. Let $A, B$ be bounded, symmetric operators on the Hilbert space $\mathscr{H}$ and assume that the product $A B$ is compact. We then have

$$
\|A B\|_{\mathscr{B}_{r}}^{r} \leqq\|B\|^{r-2}\left\|B A^{r} B\right\|_{\mathscr{B}_{1}}=\|B\|^{r-2} \cdot \operatorname{trace}\left(B A^{r} B\right) .
$$

\section{Appendix B}

Here we give the promised proof of Lemma $2.1\left(\right.$ where $\left.v_{N}:=\left|Q_{N}\right|\right)$ :

Lemma 2.1. (i) $\mathbb{E}\left(\# A^{(N)}\right)=v_{N} \cdot p$.

(ii) $\mathbb{E}\left(\# A_{\mathrm{L}}^{(N)}\right)=v_{N} p(1-p)^{v_{L}-1}+O\left(N^{v-1}\right)$.

Proof. (i) $\# A^{(N)}=\sum_{|i| \leqq N} q_{i}(\omega)$, so $\mathbb{E}\left(\# A^{(N)}\right)=v_{N} \cdot p$, since the random variables $q_{i}$ are independent and $\mathbb{E}\left(q_{i}\right)=p$.

(ii) Define

$$
\xi_{i}=\left(\prod_{\substack{j-i \mid \leqq L \\ j \neq i}}\left(1-q_{j}\right)\right) \cdot q_{i} .
$$

Then $\xi_{i}=1$ iff $q_{i}=1$ and $q_{j}=0$ for $j \neq i,|j-i| \leqq L$, i.e. if $i$ is an $L$-isolated impurity,

$$
\begin{aligned}
\mathbb{E}\left(\xi_{i}\right) & =\mathbb{E}\left(\left(\prod_{\substack{j-i \mid \leq L \\
j \neq \bar{i}}}\left(1-q_{j}\right)\right) \cdot q_{i}\right) \\
& =(1-p)^{v_{L}-1} \cdot p
\end{aligned}
$$

since the $q_{j}$ are independent.

Now, \# $A_{L}^{(N)}$ is the number of $L$-isolated impurities with distance at least $L$ from $\partial Q_{N}$, so \# $A_{L}^{(N)}$ equals $\sum_{|i| \leqq N} \xi_{i}$ up to an error of the order $N^{v-1}$. In fact, the only points that may be differently counted are those within a distance $L$ of the boundary of $Q_{N}$.

Thus,

$$
\begin{aligned}
\mathbb{E}\left(\# A_{L}^{(N)}\right) & =\mathbb{E}\left(\sum_{|i| \leqq N} \xi_{i}\right)+O\left(N^{v-1}\right) \\
& =v_{N} p(1-p)^{v_{L}-1}+O\left(N^{v-1}\right) .
\end{aligned}
$$




\section{References}

[ADH] Alama, S., Deift, P.A., Hempel, R.: Eigenvalue branches of the Schrödinger operator $H-\lambda W$ in a gap of $\sigma(H)$. Commun. Math. Phys. 121, 291-321 (1989)

[CH] Courant, R., Hilbert, D.: Methoden der Mathematischen Physik. Vol. I. Berlin, Heidelberg, New York: Springer 1968

[DH] Deift, P.A., Hempel, R.: On the existence of eigenvalues of the Schrödinger operator $H-\lambda W$ in a gap of $\sigma(H)$. Commun. Math. Phys. 103, 461-490 (1986)

[E] Eastham, M.S.P.: The spectral theory of periodic differential equations. Edinburgh: Scottish Academic Press 1973

[EK] Englisch, H., Kürsten, D.: Infinite representability of Schrödinger operators with ergodic potentials. Zeitschrift für Analysis u. ihre Anwendungen

[GS] Gesztesy, F., Simon, B.: On a theorem of Deift and Hempel. Commun. Math. Phys. 116, 503-505 (1988)

[H1] Hempel, R.: A left-indefinite generalized eigenvalue problem for Schrödinger operators. Habilitationsschrift, Univ. München, 1987

[H2] Hempel, R.: On the asymptotic distribution of eigenvalue branches of the Schrödinger operator $H-\lambda W$ in a spectral gap of $H$. J. Reine Angew. Math. 399, 38-59 (1989)

[H3] Hempel, R.: Eigenvalues in gaps and decoupling by Neumann boundary conditions. Preprint 1989. To appear in: J. Math. Anal. Appl.

[K1] Kirsch, W.: Random Schrödinger Operators. A Course. Lecture Notes in Physics 345, Berlin, Heidelberg, New York: Springer 1989

[K2] Kirsch, W.: A Continuity Property of the Density of States. Preprint

[KM1] Kirsch, W., Martinelli, F.: On the spectrum of Schrödinger operators with a random potential. Commun. Math. Phys. 85, 329-350 (1982)

[KM2] Kirsch, W., Martinelli, F.: On the density of states of Schrödinger operators with a random potential. J. Phys. A 15, 2139-2156 (1982)

[KP] Kirsch, W.: Pastur, L.A.: The Large-Time Asymptotics of Some Wiener Integrals and the Interband Light Absorption Coefficient in the Deep Fluctuation Spectrum. Commun. Math. Phys. 132, 365-382 (1990)

[Pas] Pastur, L.A.: Russ. Math. Surv. 28, 1-67 (1973)

[RS IV] Reed, M., Simon, B.: Methods of modern mathematical physics, Vol. IV: Analysis of operators. New York: Academic Press 1979

[S1] Simon, B.: Trace ideals and their applications. London: Cambridge Univ. Press 1979

[S2] Simon, B.: Schrödinger semigroups. Bull. Am. Math. Soc. (N.S.) 7, 447-526 (1982)

Communicated by B. Simon 
